\title{
Extremal Trees for the Exponential of Forgotten Topological Index
}

\author{
Akbar Jahanbani $\mathbb{D},{ }^{1}$ Murat Cancan $\mathbb{D}^{2},{ }^{2}$ and Ruhollah Motamedi ${ }^{1}$ \\ ${ }^{1}$ Department of Mathematics, Azarbaijan Shahid Madani University, Tabriz, Iran \\ ${ }^{2}$ Faculty of Education, Van Yuzuncu Yil University, Van, Turkey \\ Correspondence should be addressed to Akbar Jahanbani; akbar.jahanbani92@gmail.com
}

Received 16 September 2021; Revised 25 December 2021; Accepted 25 January 2022; Published 12 February 2022

Academic Editor: M. T. Rahim

Copyright (c) 2022 Akbar Jahanbani et al. This is an open access article distributed under the Creative Commons Attribution License, which permits unrestricted use, distribution, and reproduction in any medium, provided the original work is properly cited.

Let $F$ be the forgotten topological index of a graph $G$. The exponential of the forgotten topological index is defined as $e^{F}(G)=\sum_{(x, y) \in S} t_{x, y}(G) e^{\left(x^{2}+y^{2}\right)}$, where $t_{x, y}(G)$ is the number of edges joining vertices of degree $x$ and $y$. Let $\mathbf{T}_{n}$ be the set of trees with $n$ vertices; then, in this paper, we will show that the path $P_{n}$ has the minimum value for $e^{F}$ over $\mathbf{T}_{n}$.

\section{Introduction}

In this paper, let $V=V(G)$ and $E(G)$ be the vertex set and edge set, respectively. Let $d_{v}=d_{G}(v)$ be the degree of a vertex $v$ in graph $G$. A vertex of degree one is a pendant vertex or a leaf. A branching vertex $v$ of a tree $T$ is a vertex of degree $d_{v} \geq 3$.

Let $P=w_{0} w_{1}, \ldots, w_{q-1} w_{q}$ be the path graph with length of $r(P)=q$; if $d_{T}\left(w_{0}\right) \geq 3, d_{T}\left(w_{1}\right)=\ldots=d_{T}\left(w_{q-1}\right)=2$ and $d_{T}\left(w_{q}\right)=1$, then we called $P$ is a pendant path.

Recently, topological indices have been considered by many researchers due to their many applications in various sciences. The forgotten topological index is defined in [1] as follows:

$$
F(G)=\sum_{u v \in E(G)} d_{u}^{2}+d_{v}^{2}
$$
[2-4].

For applications of the forgotten topological index, see

Before starting a new definition, we consider the set $S=\{(x, y) \in N \times N: 1 \leq x \leq y \leq n-1\}$, and let $t_{x, y}(G)$ be the number of edges joining vertices of degree $x$ and $y$ in a graph $G$. Therefore, the new definition will be as follows:

$$
F=F(G)=\sum_{(x, y) \in S} t_{x, y}(G)\left(x^{2}+y^{2}\right) .
$$

The exponential of the forgotten topological index $F$, denoted by $e^{F}$, is defined as

$$
e^{F}=e^{F}(G)=\sum_{(x, y) \in S} t_{x, y}(G) e^{\left(x^{2}+y^{2}\right)} .
$$

Recently, the exponential topological indices have attracted the attention of many researchers. In [5], the exponential Randić index is characterized. In [6], the authors have characterized the exponential atom bond connectivity and the exponential augmented Zagreb index. In [7], the problem maximal value of trees for the exponential second Zagreb index is solved. Then, in this paper, we solve the problem with finding the minimal value of $e^{F}$ among trees.

\section{Trees with Minimum Exponential of the Forgotten Topological Index}

In this section, we will show that the path $P_{n}$ has the minimal value of the exponential forgotten topological index among all trees.

Lemma 1. Let $T$ and $T_{1}$ be the trees in Figure 1 and $A$ be a subtree of $T$. If $s \geq 3$, then $e^{F}(T)>e^{F}\left(T_{1}\right)$.

Proof. By setting $k=d_{T}(u)$, hence, we can write 


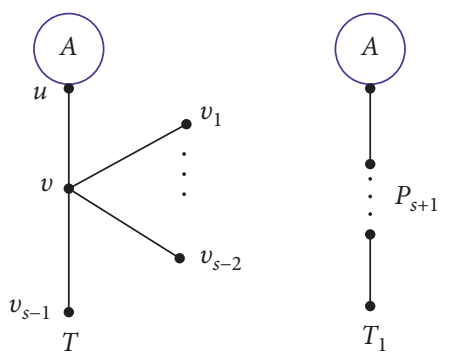

Figure 1: The trees $T$ and $T_{1}$.

$$
\begin{aligned}
e^{F}(T)-e^{F}\left(T_{1}\right)= & \left(e^{s^{2}+k^{2}}+(s-1) e^{1+s^{2}}\right) \\
& -\left(e^{4+k^{2}}+(s-2) e^{8}+e^{5}\right) \\
= & \left(e^{s^{2}+k^{2}}-e^{4+k^{2}}\right)+(s-2) \\
& \left(e^{1+s^{2}}-e^{8}\right)+\left(e^{1+s^{2}}-e^{5}\right) \\
\geq & \left(e^{9+k^{2}}-e^{4+k^{2}}\right)+\left(e^{10}-e^{8}\right)+\left(e^{10}-e^{5}\right) \\
> & \left(e^{9+k^{2}}-e^{4+k^{2}}\right)>0
\end{aligned}
$$

Lemma 2. Let $T$ be a tree with minimum value of $e^{F}$ in $\mathbf{T}_{n}$ and let $u$ be a pendant vertex $T, v \in T$. If $u v \in E(T)$, then $d_{T}(v)=2$.

Proof. Suppose $d_{T}(v)=g$ and $P$ be the largest path of $T$ and contains $v$. Let $t$ be an end vertex of $P$ and $o$ a vertex in $P$, where ot $\in E(T)$; hence, by applying Lemma 1, we have $d_{T}(o)=2$.

We continue the proof with the following two cases.

Case 1. If $d_{T}(v)=g \geq 4$.

Assuming that $T_{1}$ be the tree in Figure 2 and $A_{v}=\sum_{i=1}^{g-1} e^{g^{2}+y_{i}^{2}}$, where $y_{1}, \ldots, y_{g-1}$ are the degrees of the adjacent vertices to $v$ different from $u$. Hence, we can write

$$
\begin{aligned}
e^{F}(T)-e^{F}\left(T_{1}\right) & =A_{v}+e^{1+g^{2}}+e^{5}-\sum_{i=1}^{g-1} e^{(g-1)^{2}+y_{i}^{2}}-e^{8}-e^{5} \\
& =\left(A_{v}-\sum_{i=1}^{g-1} e^{(g-1)^{2}+y_{i}^{2}}\right)+\left(e^{1+g^{2}}-e^{8}\right) \\
& \geq\left(A_{v}-\sum_{i=1}^{g-1} e^{(g-1)^{2}+y_{i}^{2}}\right)+\left(e^{17}-e^{8}\right) \\
& >\left(A_{v}-\sum_{i=1}^{g-1} e^{(g-1)^{2}+y_{i}^{2}}\right)>0 .
\end{aligned}
$$

This contradicts the minimality of $T$.
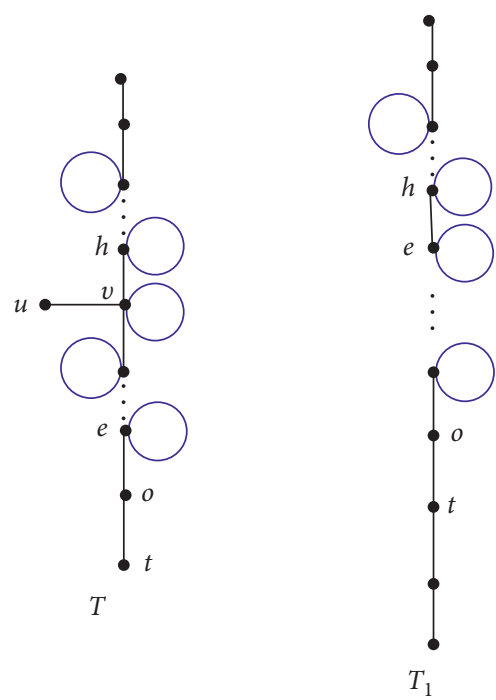

Figure 2: The trees $T$ and $T_{1}$.

Case 2. If $d_{T}(v)=3$.

Suppose $h, e \in V(T)$ and $h v, e v \in E(T)$, where $h, e \neq u$, $d_{T}(h)=a$, and $d_{T}(e)=b$. By applying Lemma 1 , we get $a \geq 2$ and $b \geq 2$. Let $T_{2}$ be the tree described in Figure 3. Therefore, we can write

$$
\begin{aligned}
e^{F}(T)-e^{F}\left(T_{2}\right) & =e^{9+a^{2}}+e^{9+b^{2}}+e^{10}-e^{a^{2}+b^{2}}-2 e^{8} \\
& =e^{9+a^{2}}+e^{9+b^{2}}-e^{a^{2}+b^{2}}+e^{10}-2 e^{8} .
\end{aligned}
$$

Here, we show

$$
f(a, b)=e^{9+a^{2}}+e^{9+b^{2}}+e^{10}>e^{a^{2}+b^{2}}+2 e^{8} .
$$

Since

$$
f(a, 2)=e^{9+a^{2}}+e^{13}+e^{10}>e^{a^{2}+4}+2 e^{8}
$$

and

$$
f(a, 3)=e^{9+a^{2}}+e^{18}+e^{10}>e^{a^{2}+9}+2 e^{8},
$$

the above inequality holds for $a \geq 2$. Therefore, for $a, b \geq 2$, we have $f(a, b)>0$. Hence, we get $e^{F}(T)>e^{F}\left(T_{2}\right)$. That is a contradiction; hence, we get $d_{T}(v)=2$.

Let $v$ be a branching vertex of degree $y$ of a tree $T$; hence, $T$ can be viewed as the coalescence of $y$ subtrees of $T$ at the vertex $v$. We call $T_{1}, \ldots, T_{y}$ are the $y$ branches of $T$ at $v$ (see Figure 4).

Definition 1 (see [5]). A branching vertex $x$ of tree $T$ is an outer branching vertex of $T$ if all branches of $T$ at $x$ except for possibly one are paths.

Lemma 3 (see [5]). A tree $T \in \mathbf{T}_{n}$ has no outer branching vertex if and only if $T \cong P_{n}$.

Lemma 4. Let $T, T_{1} \in \mathbf{T}_{n}$ be the trees in Figure 5 and $R$ be a subtree of $T, z \geq 3$ and $x=d_{T}(u) \geq 2$. Then, $e^{F}(T)>e^{F}\left(T_{1}\right)$. 


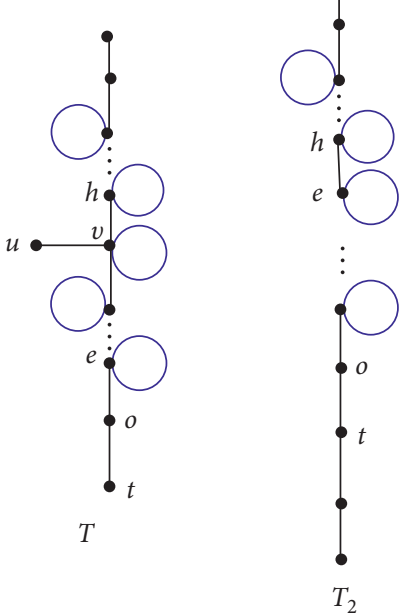

Figure 3: The trees $T$ and $T_{2}$.

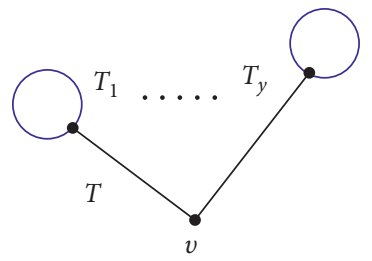

FIgURE 4: Branches of the tree $T$ at $v$.

Proof. By direct calculation, it is not difficult to see $3 \leq z \leq 8$. Here, we let $z \geq 9$; therefore, we have

$$
\begin{aligned}
e^{F}(T)-e^{F}\left(T_{1}\right)= & e^{(z+1)^{2}+4}+(z-1)\left(e^{(z+1)^{2}+4}-e^{z^{2}+4}\right) \\
& +\left(e^{(z+1)^{2}+x^{2}}-e^{z^{2}+x^{2}}\right)+\left(e^{5}-2 e^{8}\right) \\
> & e^{(z+1)^{2}+4}+\left(e^{5}-2 e^{8}\right) \geq e^{104}+\left(e^{5}-2 e^{8}\right)>0 .
\end{aligned}
$$

Lemma 5. Let $T, T_{1} \in \mathbf{T}_{n}$ be the trees in Figure 6 and $t \geq 3$. Then, $e^{F}(T)>e^{F}\left(T_{1}\right)$, where $s\left(P_{u}\right)=-2+\sum_{i=1}^{t} s\left(P_{i}\right)$.

Proof. We describe the graph in Figure 7; let $P_{t}$ be the path of length $s\left(P_{t}\right)=-2 t+2+\sum_{i=1}^{t} s\left(P_{i}\right)$. It is not difficult to see $e^{F}(T)=e^{F}\left(T_{3}\right)$. Then, by repeated of Lemma 4 , we get $e^{F}(T)>e^{F}\left(T_{1}\right)$.

Corollary 1. Let $T \in \mathbf{T}_{n}$ be a tree with a unique outer branching vertex and every pendant path has length at least 2. Then, $e^{F}(T)>e^{F}\left(P_{n}\right)$.

Proof. If $T$ has a unique outer branching vertex, hence, $T$ has the form trees in Figure 6. Let $M$ be the tree in Figure 8. If
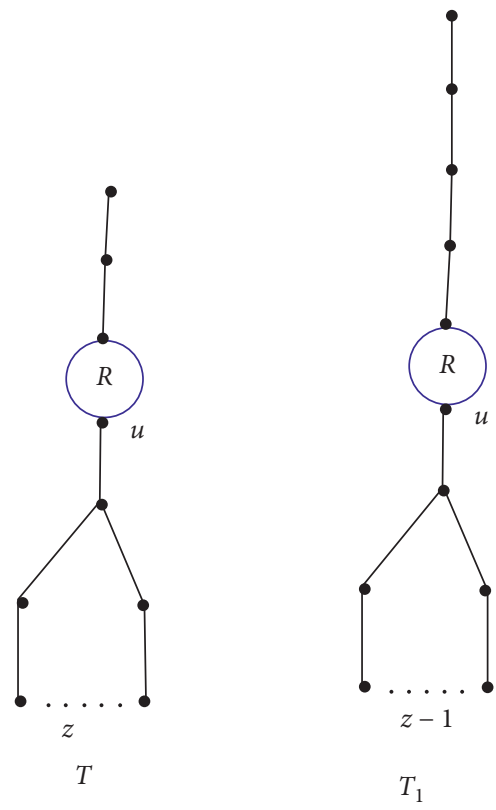

Figure 5: The trees $T$ and $T_{1}$.

$s=2$, then $e^{F}(T)=e^{F}(M)$. If $s \geq 3$, then by using Lemma 5 , we have $e^{F}(T)>e^{F}(M)$. Hence, we can write

$$
\begin{aligned}
e^{F}(T)-e^{F}\left(P_{n}\right) & =3 e^{5}+3 e^{13}+(n-7) e^{8}-2 e^{5}-(n-3) e^{8} \\
& =e^{5}+3 e^{13}-4 e^{8}>0 .
\end{aligned}
$$

Lemma 6. Let $T, T_{1} \in \mathbf{T}_{n}$ be the trees in Figure 9, such that $1 \leq d_{T}(v) \leq 3$; then, $e^{F}(T)>e^{F}\left(T_{1}\right)$.

Proof. By setting $x=d_{T}(v)$, we can write

$$
\begin{aligned}
e^{F}(T)-e^{F}\left(T_{1}\right) & =2 e^{5}+2 e^{13}+e^{9+x^{2}}-e^{5}-3 e^{8}-e^{4+x^{2}} \\
& =e^{5}+2 e^{13}-3 e^{8}+\left(e^{9+x^{2}}-e^{4+x^{2}}\right) \\
& >e^{5}+2 e^{13}-3 e^{8}>0 .
\end{aligned}
$$

Lemma 7. Let $T \in \mathbf{T}_{n}$ be the tree in Figure 10, $s \geq 1$ and $z \geq 0$; then, $T$ is not minimal in $\mathbf{T}_{n}$.

Proof. Set $x=d_{T}(u) \geq 2$, and let $T_{1}$ be tree in Figure 11 . Hence, we have $e^{F}(T)>e^{F}\left(T_{1}\right)$ if the following conditions are hold:

(1) $s \geq 1, z \geq 2$.

(2) $s \geq 4, z \geq 0$.

It is not difficult to see that our result holds for $s+z \leq 11$. Therefore, we let $z+s \geq 12$. Then, 

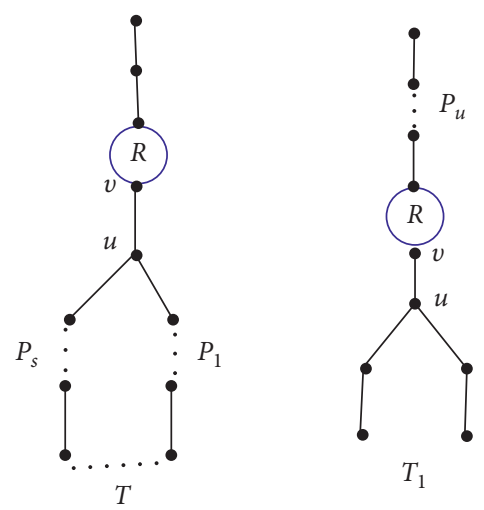

Figure 6: The trees $T$ and $T_{1}$.

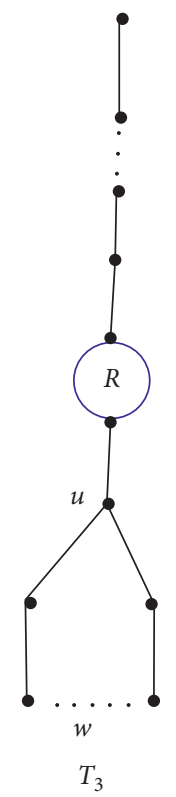

Figure 7: The tree $T_{3}$.

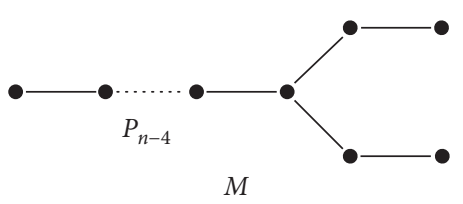

Figure 8: The tree $M$.

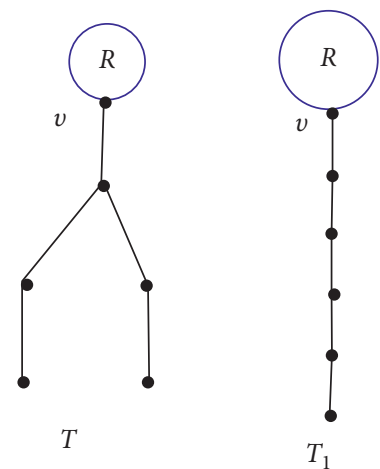

Figure 9: The trees $T$ and $T_{1}$. 


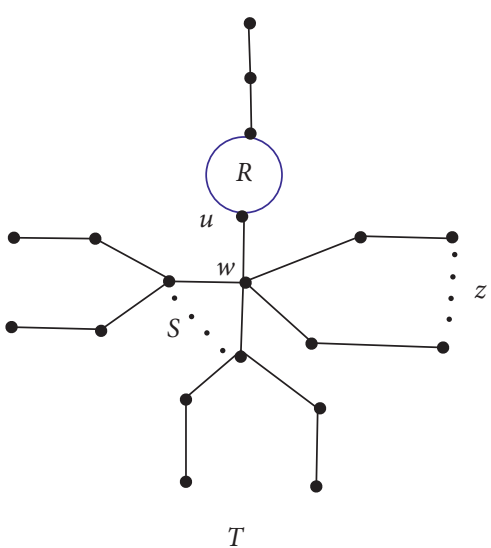

Figure 10: The tree $T$.

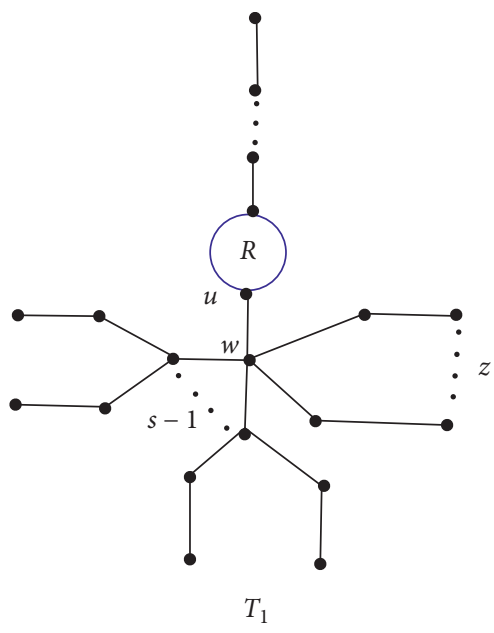

Figure 11: The tree $T_{1}$.

$$
\begin{aligned}
e^{F}(T)-e^{F}\left(T_{1}\right)= & \left(2 e^{5}+2 e^{13}-5 e^{8}\right)+(s-1) \\
& \left(e^{9+(s+z+1)^{2}}-e^{9+(s+z)^{2}}\right) \\
& +z\left(e^{4+(s+z+1)^{2}}-e^{4+(s+z)^{2}}\right) \\
& +\left(e^{x^{2}+(s+z+1)^{2}}-e^{x^{2}+(s+z)^{2}}\right)+e^{9+(s+z+1)^{2}} \\
> & \left(2 e^{5}+2 e^{13}-5 e^{8}\right)>0 .
\end{aligned}
$$

To continue the proof, we must consider the following conditions:
(3) $s=1$ and $z=0$.
(4) $s=1$ and $z=1$.
(5) $s=2$ and $z=0$.
(6) $s=3$ and $z=1$.

(7) $s=2$ and $z=1$.

(8) $s=3$ and $z=0$.

Note that, in (3), (4), and (5), we have $2 \leq d_{T}(w) \leq 3$; therefore, by Lemma 6 , we can obtain trees with the minimum value of $e^{F}$.

Here, if (6) holds, then we consider graph $T_{2}$ in Figure 12 . Hence, we can write

$$
\begin{aligned}
e^{F}(T)-e^{F}\left(T_{2}\right)= & \left(e^{5}-2 e^{8}\right)+3\left(e^{34}-e^{25}\right)+\left(e^{29}-e^{20}\right) \\
& +\left(e^{x^{2}+25}-e^{x^{2}+16}\right)+e^{20} \\
> & \left(e^{5}-2 e^{8}\right)+3\left(e^{34}-e^{25}\right)+\left(e^{29}-e^{20}\right) \\
& +e^{20}>\left(e^{5}-2 e^{8}\right)+e^{20}>0 .
\end{aligned}
$$

If (7) holds, then we consider graph $B$ in Figure 13. So, we have 

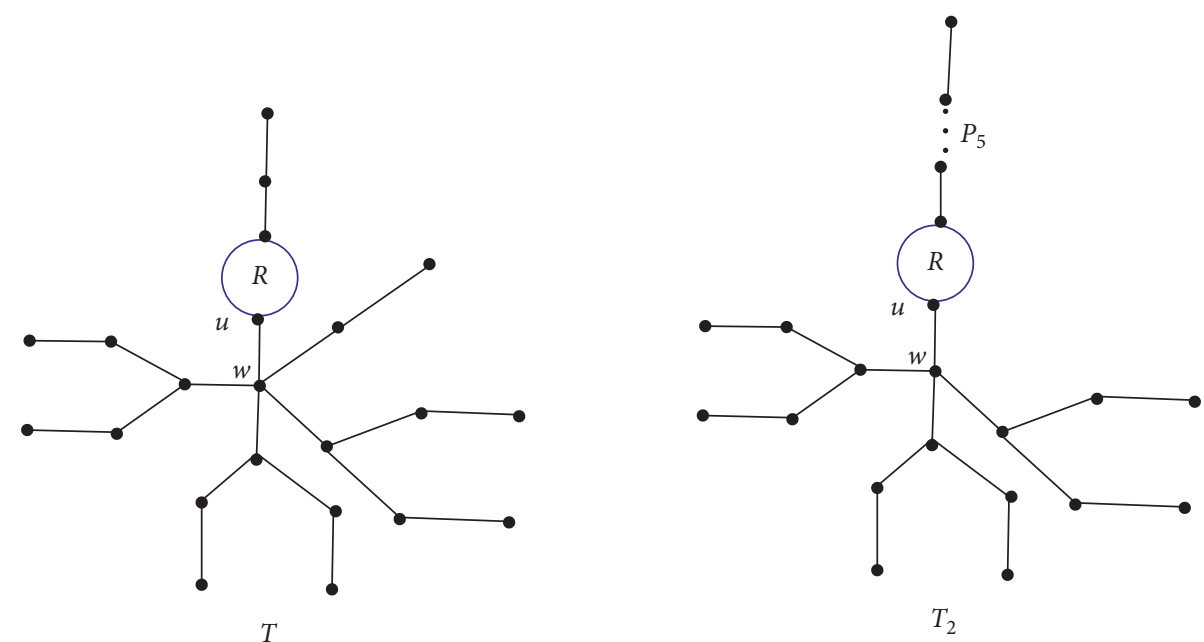

FIgURE 12: The trees $T$ and $T_{2}$.
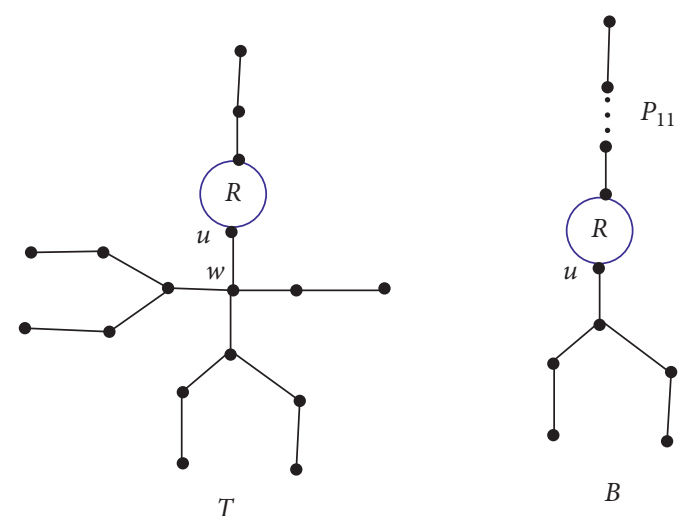

Figure 13: The trees $T$ and $B$.

$$
\begin{aligned}
e^{F}(T)-e^{F}(B)= & 6 e^{5}+4 e^{13}+2 e^{25}+e^{20}+e^{x^{2}+16}-3 e^{5} \\
& -2 e^{13}-8 e^{8}-e^{x^{2}+9} \\
= & \left(e^{x^{2}+16}-e^{x^{2}+9}\right)+3 e^{5}+2 e^{13}+2 e^{25} \\
& +e^{20}-8 e^{8}>e^{20}-8 e^{8}>0 .
\end{aligned}
$$

Finally, if (8) holds, then we consider graph $C$ in Figure 14 . Hence, we can write

$$
\begin{aligned}
e^{F}(T)-e^{F}(C)= & 7 e^{5}+6 e^{13}+3 e^{25}+e^{x^{2}+16}-3 e^{5}-2 e^{13} \\
& -11 e^{8}-e^{x^{2}+9} \\
= & \left(e^{x^{2}+16}-e^{x^{2}+9}\right)+4 e^{5}+4 e^{13}+3 e^{25} \\
& -11 e^{8}>4 e^{5}+4 e^{13}+3 e^{25}-11 e^{8}>0 .
\end{aligned}
$$

Therefore, $T$ is not minimal in $\mathbf{T}_{n}$.

Theorem 1. Let $T \in \mathbf{T}_{n}$ and $T \not P_{n}$; then, $T$ is not minimal for $e^{F}$.

Proof. By using Lemma 3, we know that $T$ has an outer branching vertex $u$. Using Lemmas 2,5 , and 6 , we let all pendant paths of $T$ have length at least 2 and $T$ has the form in Figure 15, such that $d_{T}(v) \geq 4$, and otherwise, $T$ is not minimal. If $u$ is the unique outer branching vertex of $T$, then the result obtained by Corollary 1. Otherwise, among all outer branching vertices of $T$, choose $u$ as the farthest from $u$. From Lemma 5 , we let $T$ is the form in Figure 16, such that $d_{T}\left(v_{1}\right) \geq 4$. Note that $u_{1}$ is the farthest outer branching vertex from $u$; it is clear if $T_{i}$ is not a path; then, $w_{i}$ is an outer branching vertex of $T$, and by Lemma 5 , we let $T_{i}$ have the form in Figure 17. Therefore, we get $e^{F}(T)=e^{F}(E)$, where $E$ is described in Figure 18 and $s+z=q+1$. The result follows from Lemma 7 . 

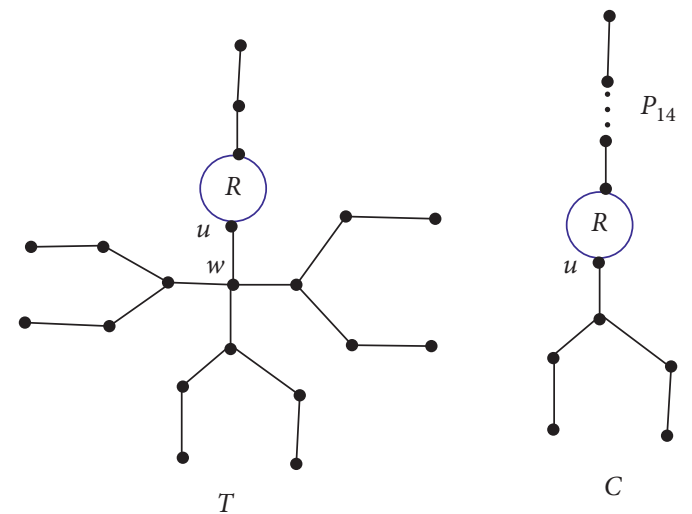

Figure 14: The trees $T$ and $C$.

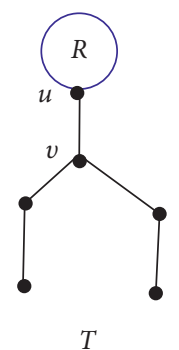

Figure 15: The tree $T$.

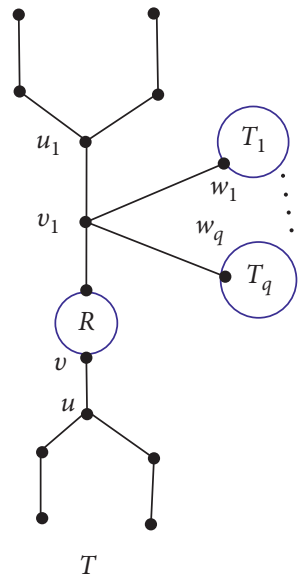

FIgURE 16: The tree $T$.

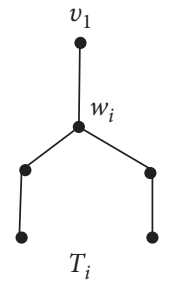

Figure 17: The tree $T_{i}$. 

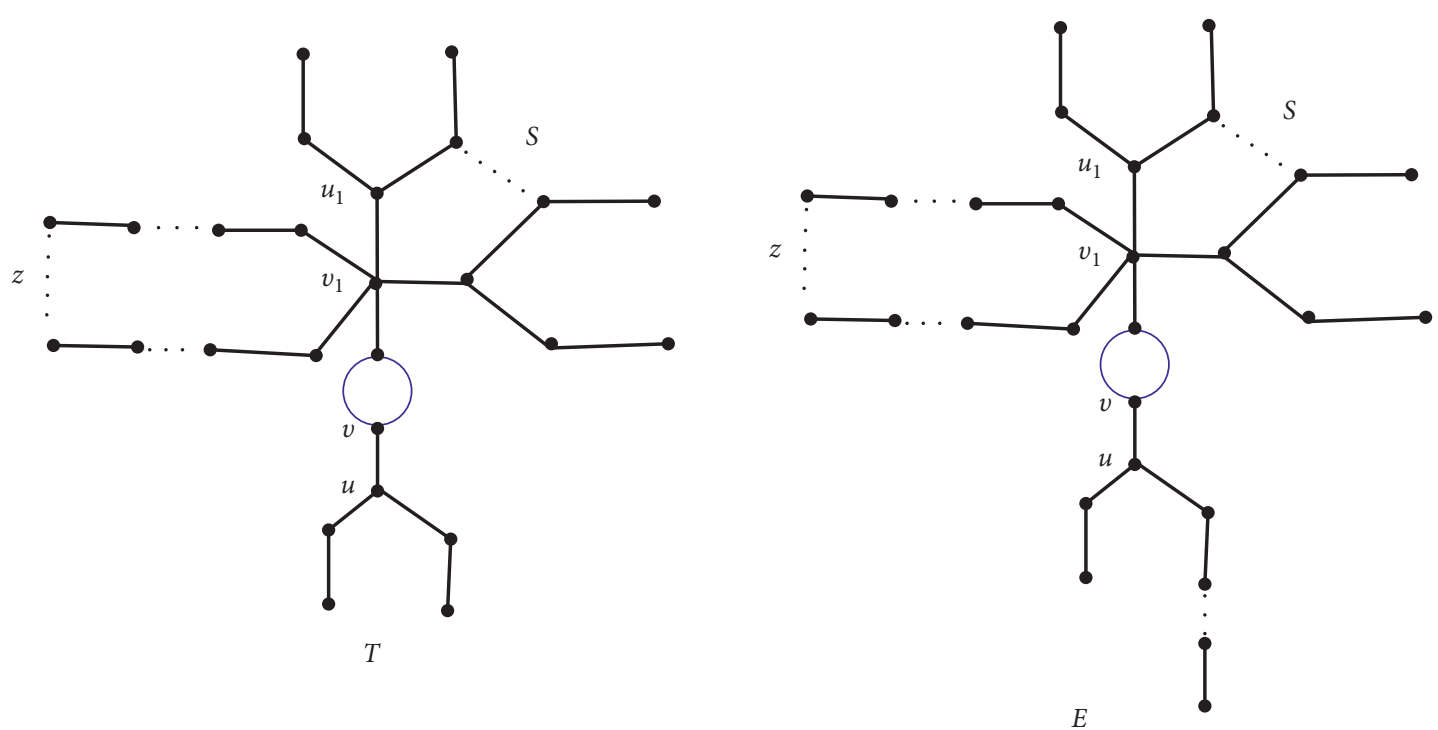

Figure 18: The tree $T$ and $E$.

\section{Data Availability}

No data were used to support the findings of this study.

\section{Conflicts of Interest}

The authors declare that they have no conflicts of interest.

\section{References}

[1] B. Furtula and I. Gutman, "A forgotten topological index," Journal of Mathematical Chemistry, vol. 53, no. 4, pp. 11841190, 2015.

[2] I. Gutman and N. Trinajstić, "Graph theory and molecular orbitals: total $\varphi$-electron energy of alternant hydrocarbons," Chemical Physics Letters, vol. 17, no. 4, pp. 535-538, 1972.

[3] A. Jahanbani, "On the forgotten topological index of graphs, discrete mathematics," Algorithms and Applications, vol. 13, no. 5, Article ID 2150054, 2020.

[4] A. Jahanbani, "New bounds for degree sequence of graphs," Computer Science Journal of Moldova, vol. 80, pp. 188-203, 2019.

[5] R. Cruz, J. Monsalve, and J. Rada, "Trees with maximum exponential randić index," Discrete Applied Mathematics, vol. 283, no. 3, 2020.

[6] R. Cruz and J. Rada, "The path and the star as extremal values of vertex-degree-based topological indices among trees," MATCH Communications in Mathematical and in Computer Chemistry, vol. 82, pp. 715-732, 2019.

[7] M. Zeng and H. Dengy, "The maximal tree with respect to the exponential of the second zagreb index," 2020, https://arxiv. org/abs2006.08892v1. 